\title{
A Design for a Picture Archiving Communication System Central Storage Facility
}

\author{
Gilbert E. Houtekamer, Leonard J.N. Franken, Gerard L. Reijns, and Wim J.J. Stut, Jr
}

\begin{abstract}
A Picture Archive and Communication System (PACS) as it is to be used in a Hospital will consist of a number of workstations, data acquisition stations and output stations, connected to a central storage facility. In this paper the architecture of the central storage facility is studied, and an implementation is proposed. Simulation studies have been performed on the proposed implementations, showing their feasibility. The simulation studies show that the central storage facility can store over 500 sets on a daily basis, while simultaneously handling the review and output activity associated with such a production. On the average, each set is assumed to consist of 3 images of 4 Mbyte each. Simulation results are presented for a number of alternative systems. Special attention is given to the implications of prefetching on the protocols to be used in the PACS system. It is shown that prefetching, in particular in combination with a pipelined system, can yield significant performance benefits. We believe that prefetching of images is a prerequisite for good system performance. The implementation assumes that a high-speed communication protocol is used to interconnect the PACS components. The paper will not go into details on the network considerations. The central storage facility is designed using a general purpose System/370 machine, with the peripherals commonly used for such machines. This implies that it can be realized with currently available equipment. Several alternative implementations using interconnected workstation based systems are suggested.

Copyright $\bigcirc 1991$ by W.B. Saunders Company
\end{abstract}

KEYWORDS: PACS, image storage, standards, ACRNEMA, prefetching strategies for PACS.

$\mathbf{W}$ ITH THE advent of modern imaging techniques in hospitals, the need for an electronic data (image) storage facility becomes more pressing. The images created by devices such as conventional screen-film radiographic $\mathrm{x}$-ray imaging systems and nuclear magnetic resonance (NMR) scanners are now archived in hardcopy form. This has many drawbacks: the

From the Delft University of Technology, Department of Electrical Engineering, Delft, The Netherlands, and BAZIS Central Development and Support Group Hospital Information Systems, Leiden, The Netherlands.

Address reprint requests to $\mathrm{Dr}$ G.E. Houtekamer, Delft University of Technology, Department of Electrical Engineering, PO Box 5031, 2600 GA Delft, The Netherlands.

Copyright 11991 by W.B. Saunders Company

0897-1889/91/0402-0004\$03.00/0 copy can be on one place only, it requires a lot of storage space, and the copies can easily get lost.

An electronic mass storage system (archive) could overcome most of the these problems, if the data acquisition stations are connected to it. Images can then be viewed with workstations connected to the archive. If the images are stored in such an electronic central storage facility, it also becomes feasible to use image restoration and enhancement techniques. The entire network of acquisition stations, workstations, and central storage facility is called a Picture Archive and Communication System (PACS). In this article we will consider the architecture of the central storage facility, taking into account both the volume and speed requirements for image storage and retrieval. Because of the huge volume of data (roughly 6 Gbytes a day), and the long retention period (10 years, half a year on-line), the images must be stored on an optical or magnetic (tape) archive. Such archives, however, do not normally support the high data rates, and short seek times, that are required for the workstations. It is therefore proposed to use a magnetic disk pool as a buffer between the archive and the communications network. Figure 1 depicts such a system.

Acquisition stations represent the equipment in the hospital used to create images. These stations include $x$-ray imaging systems, NMR devices, computer tomography, and ultra sound stations. The size of the images created will vary widely between the modalities, from as little as $128 \times 128 \times 8$ bits for ultrasound scans to $2048 \times 2048 \times 12$ for scanned $x$-ray images. In the article the notion of sets is used to denote a set of images with a total of size 12 Mbyte, for example 2 to 4 scanned x-ray images of 4 Mbyte each. It is assumed that each acquisition station is equipped with enough memory to store one such set of images.

Work stations are used by the radiologists for the primary diagnosis. A review session consists of the inspection of new and old images for a patient. After inspection of the images, the 


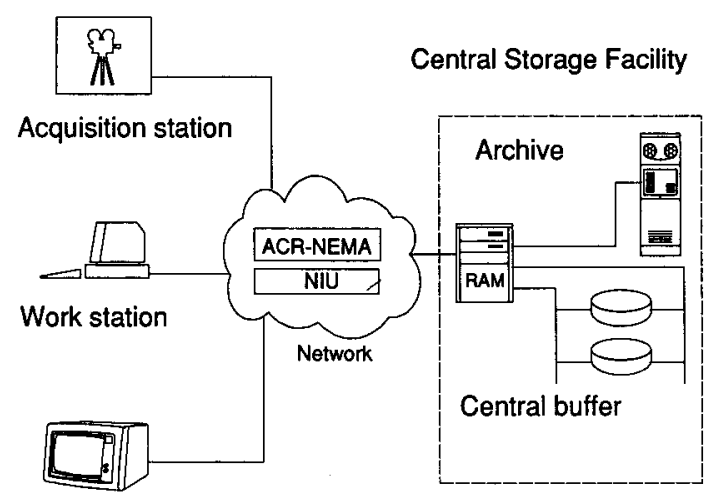

Output Only Station

Fig 1. PACS system studied, with acquisition stations, work stations and output only stations to create and view images on the left. On the right the central storage facility is shown, with the disk pool.

radiologist will write some conclusions to be stored with the images. For these stations, the images have to be presented at their maximum resolution.

Output Only Stations are used by other specialists to view images. Normally, the images viewed are the ones that have been created or used for diagnosis earlier on the same day. The images might be presented at a lower resolution than on the workstations, to save network load and to reduce the cost of the output stations. In this study it was assumed that the images are handled, but not necessarily presented, at full resolution.

The acquisition stations, workstations, and output only stations will be referred to collectively as network stations in this article.

The use of an American College of RadiologyNational Electrical Manufacturers Association (ACR-NEMA)-like network protocol is assumed. ${ }^{1}$ The ACR-NEMA committee has defined a network protocol conforming to the layered International Organization for Standardization-Open Systems Interconnect interface model. A description of the network protocol is beyond the scope of this article. It suffices to know that the network protocol implements point-to-point interfaces with a handshake mechanism. Currently, special purpose interfaces are envisioned that support an $8 \mathrm{Mbyte} / \mathrm{s}$ data rate on a point-to-point connection. As will be described later, we did not strictly adhere to the requirements of the ACR-NEMA standard, but rather used it as a guide-line on the network speed.

The central storage facility controls the archive and central buffer. The central buffer is used to store images that have been created during the last 24 hours, since these images are most likely to be retrieved for inspection by the workstation and output only station users. This paper will focus on the design of the central storage facility, considering parameters such as the size of the processor in the system, the (real) memory requirements of the processor in the system, the speed of the archive, and the network protocol to be used.

The structure of the paper is as follows. Section 2 describes the workload of the system, as it is known from measurements and observation in hospitals. Section 3 presents some of the design considerations for the PACS central storage. Section 4 describes the two implementations of a PACS systems that we analyzed: one using a tape (cartridge) archive, and one using an optical disk archive. Section 5 describes the performance results obtained from a simulation study. Section 6 proposes some alternative architectures that can be considered for the PACS central storage facility. Finally section 7 summarizes the paper.

\section{PACS WORKLOAD}

It is difficult to estimate the workload on a PACS system if no system is available to take measurements from. Workload estimates must be based on current hospital procedures. This workload will inevitably change when a PACS system is getting into use. One would expect a radiologist or specialist to ask for more information if the PACS system can provide it quickly and conveniently. It is a well known phenomenon that new or better facilities attract extra workload.

For the time being, however, we assume that observation data from the current hospital procedures can be used. To estimate the workload for the acquisition stations, the current production from acquisition stations can be used. Since this number is chiefly determined by the number of patients in a hospital, it is not likely to change very much with the introduction of a PACS system. The total daily production for a medium sized hospital is about 500 sets, a set 
being defined as a group of 2 to 4 images of 4 Mbyte each. The distribution is heavily skewed, however, yielding a busiest hour of about 70 sets. Figure 2 shows a sample production distribution for the acquisition stations. ${ }^{2}$ It will be obvious that the PACS system must be designed to meet the demand in the busiest hour. Also, note that the number of sets mentioned here and the number of images per set will differ significantly between hospitals. The numbers provided here, and the other workload figures presented in this paper, should be considered as estimates only. It cannot be stressed enough that the workload in each hospital will be different, depending on the hospital size, specialization and operational procedures.

The workstations shown in Fig 1 are used by radiologists for primary diagnosis, using the images created at the acquisition stations. Radiologists tend to review these images in batches, to use their time as efficient as possible. A review session will then start when a number of sets are available for interpretation. In our study, it was assumed that the review process starts as soon as 10 sets are available, and that in each review session precisely 10 new sets were analyzed. In addition is was assumed that on the average one old set was inspected with each new set, in order to detect changes from previous observations. As is to expected, the review process (Fig 3) peaks just after the acquisition. ${ }^{2}$

The old sets required in the review session must be loaded from the archive. Since the review session is scheduled to start only when a number of sets are available, the old sets can be prefetched from the archive. The prefetch can

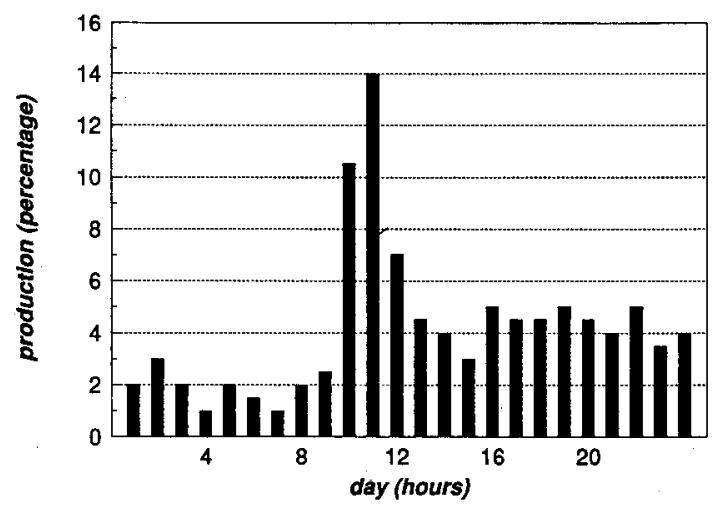

Fig 2. Distribution of the production workload over the day.

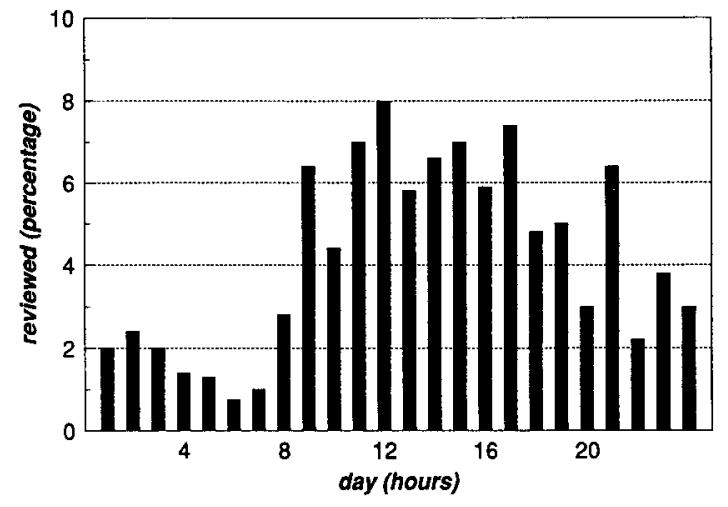

Fig 3. Distribution of the review (primary diagnosis) load over the day.

be automatic (under control of the central system and/or hospital information system), or manual (under control of an assistant). In our model it is assumed that all old sets can be prefetched from the archive: the load process can start as soon as the new set is available, ie, the system does not have to wait until the set is actually requested for inspection at the workstation.

The total daily workload for the workstations amounts to 12 Gbytes a day (500 old sets and 500 new sets). For each set, the radiologist will add some consultations to be stored (about 380 bytes $\left.^{3}\right)$. The data volume of the comments is negligible. In this article, the comments are thought to be stored (logically) with the images. In a real implementation, they may be stored (physically) in the hospital information system separately from the images.

Not much information was available on the requests made from the Output Only Stations. It was assumed in the simulations described below that the specialists use the Output Only Stations to inspect data that was collected earlier the same day. Timing estimates will be provided for the response time to load a set of images from the central buffer, and to load a set from the archive (ie, a set that was not yet prefetched). Just as for the review sessions, the performance for the specialists can be improved significantly if an assistant prefetches the images from the archive into the central buffer.

Finally, a word on the archive itself. With the assumed acquisition rate of 500 sets a day, the daily production amounts to $500 * 3$ (images/ set) $* 4$ Mbyte $\approx 6$ Gbyte. If the information is 
to be kept on-line for half a year, the archive size needs to be $6 * 180 \approx 2,000$ Gbyte. This is larger than the largest optical disk and cartridge jukebox configuration. This number will differ between hospitals, depending on the number of patients and the resolution of the images. Other authors mentioned a figure of 4 Gbyte per day, assuming 4 Mbyte images. ${ }^{4}$ They also noted that much smaller archives can be used if lower image resolutions are used. Unfortunately there is little clinical experience as to what resolutions are acceptable for the various analyses.

From this size consideration it can be concluded that it will not be feasible for some time to store all information over the last 5 or 10 years on-line; operator assistance will be required to use old sets. Further research will be required to establish a reasonable retention period for data in the on-line archive, based on set reference patterns. As an alternative to an electronic archive, one could also store the images on conventional film for long term storage.

\section{DESIGN CONSIDERATIONS}

A prime performance parameter for the PACS system is the time required to access a set of images stored in the central storage facility. An average set is assumed to consist of three images, and to require 12 Mbyte total. If the archive consists of tape cartridge units equipped with a robot, ${ }^{5}$ the load time for a set would be about $40 \mathrm{sec}$ ( $10 \mathrm{sec}$ to load a tape, $30 \mathrm{sec}$ to locate the information on the tape), while the data transfer would require about $4 \sec (3$ Mbyte/s). If the archive consists of optical disks, the load time would be about $8 \mathrm{sec}$, and the data transfer time $12 \mathrm{sec}$. These response times are too high to be acceptable.

Therefore, a central storage facility is proposed that consists of a tape cartridge or optical storage system, supplemented with a so-called central buffer, consisting of (magnetic) disk drives. These magnetic disks are used to temporarily store sets from acquisition stations, and to prefetch sets from the archive. Magnetic disk drives provide a very short seek time ( 5 to 20 $\mathrm{ms}$ ), and a relatively high data rate (3 to 4 Mbyte/s). A higher throughput can be achieved by using several disks in parallel, as will be discussed momentarily.
Most references to sets in a PACS system are to sets created the same day: A review session and an inspection on an output only session will normally occur on the same day as the acquisition. This implies that a set created by an acquisition station should be kept on magnetic disk at least for 1 day, to avoid unnecessary accesses to the archive. In the system described, a set is archived as soon as the review session is completed, such that the entire set and the comments made by the reviewing radiologist can be stored on the same archive cartridge or optical disk. Once the interpretation has been completed, the set will not be updated any more, although some provision must be made to add additional notes later on (in the PACS or the Hospital Information System [HIS] that connects to the PACS).

The use of the central buffer as a 1 day buffer also has the advantage that the archive process (writing to the archive) is not time-critical: the archive functions can run as a background task during periods of low activity, eg, during the night. This leaves the archive available during day hours to prefetch old sets required for review sessions: as sets arrive from the acquisition stations, the corresponding old sets can be prefetched before the actual requests from a workstation arrive. As described above, such a prefetch procedure avoids unnecessary delays at the workstation.

The information from a set can be stored in many ways in the central buffer: A set could be placed entirely on one disk, it could be spread over disks on a per-image basis, or it could be spread over all disks in smaller increments. Spreading (the images in) a set over disks on a per-image basis has the advantage that a set can be loaded quicker: Several disks can operate in parallel to load images of the set (one image per disk). Spreading the images in a set over all disks will not provide more performance benefits as compared to spreading on a per set basis, since most disk subsystems support only two or four concurrent data transfers at any given time. This number corresponds to the average number of sets in an image, so spreading over disks on a per image basis will provide most of the performance benefits that can be achieved from parallelism in such a subsystem. Note that this conclusion is based on the particular disk sub- 
system implementation studied, and on the workload assumptions (average number of sets per image). See Gibson for a full discussion of possible parallel disk architectures in a more general context. ${ }^{6}$

The transfer of images between the central storage facility and the network involves large amounts of data. It is therefore essential to minimize the amount of processor storage required for each operation. In the system proposed, it is assumed that each station on the network has sufficient local memory to store a full set of images. If this is the case, a handshake process between network station and central storage facility can be used such that no more than, eg, 1.2 Mbyte of buffer area is required for each process. This buffer area can be used to store several blocks that must be transferred from disk to the network. A large blocksize is advantageous since it allows for longer transfers from disk, that are more efficient: Each transfer will start with a seek to position the head, and a rotational delay to reach the information. Once the data transfer starts, no overhead is involved.

A small blocksize is advantageous since the information transfer to (or from) the network station can start earlier. The particular buffer size of 1.2 Mbyte as proposed corresponds to two 600 Kbyte disk blocks, the maximum size for the disk type studied. It allows for efficient disk transfers, while it permits data to be handed to the network station well before (after $600 \mathrm{Kbyte}$ ) the entire image (or set) is read from disk. This is important, since it means that some concurrency can be achieved in the central storage facility and network station processing. In the simulation studies the impact of the processor storage size and the handshake strategy will be discussed.

There are some prerequisites for this method to work, however. Some communication protocols assume that a full message (image in the ACR-NEMA context) must be present in storage before a transmit can start, and vice versa that a full image must be received before it can be processed. The pipeline approach as presented above assumes that a partial image can be processed; ie, a transmit from the central storage facility is assumed to start as soon as a large block has been read from disk. Such a pipeline has some performance advantages, but it may not be feasible with the ACR-NEMA and network protocols to be used.

The problems that may occur with the proto$\mathrm{col}$, and ways to get around them, have not been investigated yet. It seems natural that in real hospital implementations of the system other industry standards will be used in addition to the ACR-NEMA standard. For example, the Standard Product Interconnect (SPI) standard jointly developed by Siemens (Erlangen, Germany) and Philips (Best, The Netherlands) allows systems to use the Ethernet (Xerox Corp) and Fiber Distributed Data Interface carriers, and the Transmission Control Protocol/ Internet Protocol standards for the network handling. ${ }^{7-9}$ SPI is defined as an extension of the ACR-NEMA standard, ie, the normal ACRNEMA protocols are used to specify the image and set layout, ie, the medical aspects of the message. Such solutions allow for the use of cheap, off-the-shelf hardware, greatly reducing the costs of PACS implementations. On the other hand, they may preclude optimizations that are not supported by the standard protocols used.

If these problems preclude the full use of spreading and pipelining, spreading can still be used to transfer information from the disks in the central buffer to the processor storage, allowing for a quick transfer of data to the processor storage.

In the next section a system configuration will be proposed based on the analysis presented so far. It will be assumed that both spreading and pipelining can be implemented.

\section{IMPLEMENTATIONS SELECTED}

In the design of the system only current technology is used, to make the design as realistic as possible. It is anticipated that the total capacity of the system can increase as newer equipment becomes available. For the disk subsystem, the technology from the IBM 3380 mainframe disks was used to set the parameters. These disks are available from many manufacturers, in various storage capacities. ${ }^{10}$ The parameters from these disks that were used in the simulations are summarized in Table 1.

For the tape cartridge archive, the Memorex Telex (Memorex Telex Corp,) cartridge robot 


\begin{tabular}{lc}
$\begin{array}{l}\text { Table 1. Characteristics for an IBM 3380D Disk Subsystem } \\
\text { With 16 Disks, as Used for Our Simulations }\end{array}$ \\
\hline Capacity (4 Kbyte blocks) & $530 \mathrm{Mbyte}$ \\
& $132 \mathrm{images}$ \\
Seek time (average) & $16 \mathrm{~ms}$ \\
Data rate & $3 \mathrm{Mbyte} / \mathrm{s}$ \\
Concurrent transfers/subsystem & 2 or 4 \\
Disks/subsystem & 16 \\
\hline
\end{tabular}

was used as a model, ${ }^{5}$ in combination with the IBM 3490 tape cartridge unit specifications. ${ }^{11}$ The cartridge robot can manage a rack with 550 to 5,000 cartridges, and load any of these cartridges in one of the units. Once loaded, the cartridge must be searched for the set requested (in case of a read). Once the set has been located, the data transfer can occur at a relatively high speed. The characteristics are summarized in Table 2 below.

The performance characteristics from the optical archive change significantly from the tape cartridge system. The optical archive can search information quickly, but has a much lower data transfer rate. One of the fastest systems currently available is the Kodak System 6800 (Eastman Kodak Company, Rochester, NY). This system supports 50 to 150 disks with a 6.8 Gbytes capacity per disk. ${ }^{12,13}$ The essential characteristics are summarized in Table 3.

It should be noted here that the selection the disk, tape robot and optical storage systems here does not imply that these particular systems are best suited for this task. Rather, they should be considered as representative examples of what is currently on the market.

The network interface in the central system is assumed to operate at its maximum specified data rate of $8 \mathrm{Mbyte} / \mathrm{s}$, the data rate proposed for the current ACR/NEMA standard. Any delays that may occur as a result of protocol overhead are ignored in the simulation model, as described in the previous section.

Table 2. Characteristics for a Tape Cartridge System With Robot

\begin{tabular}{lc} 
Capacity per cartridge & 300 Mbyte \\
Cartridges per system & 550 to 5000 \\
Robot load time & $10 \mathrm{~s}$ \\
Search time & $40 \mathrm{~s}$ \\
Data rate & $4.5 \mathrm{Mbyte} / \mathrm{s}$ \\
Concurrent transfers/subsystem & 2 \\
Cartridge units/subsystem & 8 or 16 \\
\hline
\end{tabular}

Table 3. Characteristics for the Kodak System 6800 Optical Storage System

\begin{tabular}{lc}
\hline Capacity per disk & 6.8 Gbyte \\
Disks per system & 50 to 150 \\
Robot load time & $6.5 \mathrm{~s}$ \\
Search time & $0.7 \mathrm{~s}$ \\
Data rate & $1 \mathrm{Mbyte} / \mathrm{s}$ \\
Concurrent transfers/subsystem & 1 \\
Read/write units/subsystem & 1 to 3 \\
\hline
\end{tabular}

\section{PERFORMANCE RESULTS}

A simulation model of the system configuration discussed in the previous section was built using the Research Queueing Package (RESQ) simulation package. ${ }^{14}$ RESQ is a discrete event simulation program, specifically designed to simulate computer systems and networks. The simulation model represents all performance characteristics of the equipment as outlined in the previous sections. It also takes into account the impact of limited storage in the processor, in relation to various methods to transfer to the data over the network. These configuration options are described in more detail below.

The simulation model is split up into several submodels, representing the disks in the central buffer, the processor storage, the archive and the network interface. The central buffer is simulated with 16 disks, with one controller that has two to four independent data paths. Various ways of placing the data are simulated, as indicated below. Two versions of the archive model are simulated, representing the optical and tape units described in the previous section. The network interface logic is not simulated; it is simply considered as a device that can transfer data at $8 \mathrm{Mbyte} / \mathrm{s}$.

Several job-types are used in the simulation, representing the acquisition, archive, workstation, and output-only station workload. The prime workload parameters that have been used in the system simulations have been presented in the workload section before. To determine the maximum throughput that can be handled with the central buffer implementation proposed, simulations have been performed on the central buffer only (neglecting the archive). For these simulations, a review workload was used, with 4 to 60 review sessions per hour, amounting to the transfer of 80 to 1200 sets per hour from the central storage facility to the workstations. A Poisson arrival process was 


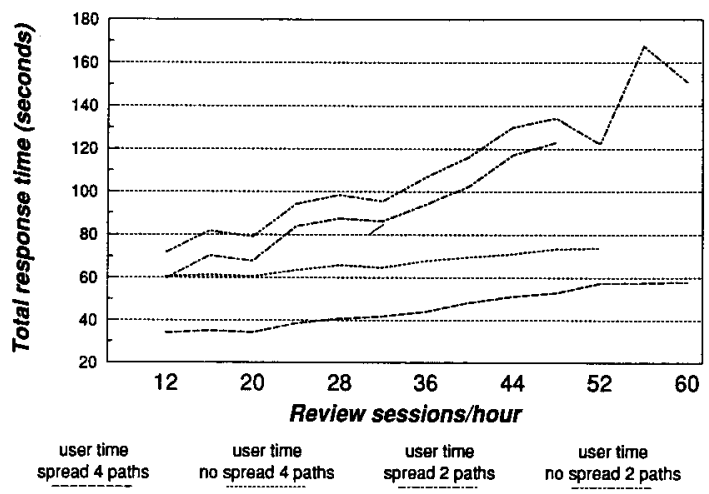

Fig 4. The total time to send 20 sets from the central storage facility to a workstation. The response time reported includes the network delays.

assumed for all processes: for the review sessions, for the acquisition load and for the output only stations.

Figure 4 shows the results from these simulations. Figure 4 shows that the best response times are achieved for systems with 4 paths between the disk subsystem and the processor: more paths mean more concurrent transfers and thus a higher throughput. It also shows that spreading the information is beneficial, since spreading allows for the best use of multiple storage paths.

For the system as proposed up to about 50 review sessions $(1,000$ sets) can be handled per hour with both the 2 and 4 path configurations. Note that a review session was defined as a primary diagnosis session in which 20 sets are studied for each patient. One thousand sets per hour amounts to an average sustained data rate of about $3.3 \mathrm{Mbyte} / \mathrm{s}$, or a $40 \%$ utilization of the network. The 4 path configuration can support higher loads; the performance will then be limited by the assumed network speed.
For real use of the system, results with a mixed workload from all network stations are more interesting. In the simulation of the system as described above, the following load processes are considered: (1) acquisition process: production of new sets and retrieval of one old set with each new set from the archive; (2) workstation process: review old and new sets; (3) output only process: view sets; and (4) archive process: archive sets that have been reviewed.

For the base simulation run, only the acquisition and workstation processes are included, representing the activity around 10 a.m. (Figs 2 and 3). The other relevant system parameters are summarized in Table 4 below. Each alternative listed in the right column of Table 4 differs in only one parameter from the base situation A.

The simulation results include the time to store an image from an acquisition station, the wait time at a workstation to receive all sets needed for a complete session (10 old and 10 new sets), and the time to retrieve an image from the archive.

Table 5 shows these results. The Acquisition Job Image Time is the average time required to store a single image offered by an acquisition station in the central buffer. The Work Job User Time is the total time required to transmit 10 old and 10 new sets from the central buffer to the radiologists workstation. It is assumed that all old and new sets are already present in the central buffer (ie, that no archive activity is required). The Archive Random Read Access time is the response time for a request to load one set of images from the archive to the processor storage. Note that this time excludes the transfer to the output only station, and

Table 4. Parameters Used in the Simulations

\begin{tabular}{llll}
\hline \multicolumn{1}{c}{ Component } & \multicolumn{1}{c}{ Base Situation A } & Alternative \\
\hline Central buffer & 16 disks, 4 paths & $\mathrm{B}$ & 16 disks, 2 paths \\
processor storage & Infinite & $\mathrm{C}$ & 12 segments of $0.6 \mathrm{Mbyte}$ \\
Pipeline & Cylinder $(0.6 \mathrm{Mbyte})$ & $\mathrm{D}$ & Image (4 Mbyte) \\
Spread set? & yes & $\mathrm{E}$ & no \\
Archive & optical & $\mathrm{G}$ & tape \\
Load: & Acquisition: 70 sets/hour & $\mathrm{F}$ & Acquisition: 70 sets/hour \\
& Workstation: 4 sessions/hour & & Workstation: 4 sessions/hour \\
& Output Only Stations: 0 sets & & Output Only Stations: 50 sets/hour \\
& Archive: 0 sets & & Archive: 70 sets/hour
\end{tabular}


Table 5. Simulation Results for the Configurations Defined in Table 4

\begin{tabular}{cccc}
\hline $\begin{array}{c}\text { All Times } \\
\text { in s }\end{array}$ & $\begin{array}{c}\text { Acquisition Job } \\
\text { Image Time }\end{array}$ & $\begin{array}{c}\text { Work Job } \\
\text { User Time }\end{array}$ & $\begin{array}{c}\text { Archive Random } \\
\text { Read Access }\end{array}$ \\
\hline A & 2.2 & 37 & 22 \\
B & 3.4 & 64 & 22 \\
C & 2.2 & 36 & 24 \\
D & 3.6 & 52 & 22 \\
E & - & 61 & 26 \\
F & 2.2 & 40 & 25 \\
G & 2.1 & 37 & 44 \\
\hline
\end{tabular}

excludes the time to unload the drive and return it to the robot.

Table 5 shows that both the acquisition and work jobs delays are acceptable in most configurations. The Acq Job Image Time is the time required to store an image in the central buffer, and thus depends on the network and central buffer performance. In the system as studied, the central buffer was implemented with two paths between disk subsystem and processor (situation B), or with four paths (other situations). The simulation results indicate that the four path configuration provides a $2.2 \mathrm{~s}$ response time, and that the two path configuration provides a $3.4 \mathrm{~s}$ response time. The increased response time in situation $B$ is caused by path contention, while the response time in the four path situation is determined by the data rates of the disks and network. The amount of processor storage (situation $\mathrm{C}$ ), the archive type, and a somewhat higher system load do not change the response time. The pipeline mechanism used is important: Situation D describes a system in which an entire image is first read into the processor storage, and subsequently transferred to disk. This is significantly slower than the overlapped processing that is used in the other situations: as soon as 600 Kbyte of an image is present, that part is transferred to disk. To conclude, the disk data rate, the number of paths to the disk subsystem, and the pipeline mechanism are important for the acquisition time.

The results for the workstations are similar, since they also describe the transfer of information between the disks and the network stations, be it in the other direction. The same considerations apply for the acquisition stations: four paths are important, and pipelining should be used. For the workstation time, data is also available for situation $\mathrm{E}$, that describes a model in which images from a set are not spread over several disks. Rather, they are all stored on the same disk. This causes extra delay, since the images cannot be read in parallel into the processor storage. Spreading thus provides a significant performance advantage.

The archive (read) access time is almost constant for the situations A to $F$, that all use an optical archive. The archive is much slower than the other system components, which makes the overall system configuration not very important for the archive access time. The archive access is faster for the optical archive than it is for the tape cartridge archive, since the tape subsystem suffers from a very large search time. This search time offsets the advantage of the higher data rate that the tape subsystem offers. It should be noted, however, that the tape subsystem performs the archive write function faster: 9.6 seconds per set versus 29.6 seconds for the optical archive. Tapes are more efficient than optical disks for this archive operation since all sets can be written to the same tape: the load and search time is minimal, and the data rate advantage can be exploited fully. Read operations from the archive (the access time described above) are to a random tape of optical disk, that needs to be fetched from the archive. Each archive read will thus include the robot and search delays.

\section{ALTERNATIVE ARCHITECTURES}

In the implementation discussed above all logic related with the central storage facility is concentrated in a single computer system. This may cause (cost) problems with the central system, since the system must support high data rates: up to $8 \mathrm{Mbyte} / \mathrm{s}$ (burst) to the network, up $12 \mathrm{Mbyte} / \mathrm{s}$ (bursts) to the disks, up to 4.5 Mbyte/s to the tape archive, and/or up to 1 Mbyte/s to the optical archive.

It is possible to distribute the central buffer function over a few smaller disk systems, that each support one concurrent disk transfer, and each use their own network interface to transfer data to the network stations. A separate supervisor system could then support the archive, and control the disk systems. An industry standard 
connection could be used to connect the disk systems and the supervisor system for command and data transfers between these systems (in particular to retrieve data from the archive to the disk buffers).

Figure 5 depicts such a system. Requests for image retrieval or storage must be addressed to the supervisor system, which assigns the request to one of the disk systems. The advantage of this approach is that industry standard equipment can be used for the disk and supervisor systems, eg, fast personal computers (PCs) with a large harddisk. The problems are that additional complications will arise at the protocol level: A network station would have to request service from a supervisor station, that must assign a (slave) disk system. The overall response time could be roughly the same as for the approach presented before, if fast disks and fast interfaces are used in the disk and supervisor systems. It should be noted in this context that although PC busses like the PS/2 Microchannel (MCA) (IBM Corp) bus and the EISA bus support high data rates theoretically, most controllers are unable to make use of these high data rates.

Yet another approach would be to use special purpose high speed disks and disk arrays that become available now. Compaq (Houston, TX) provides with a disk controller that can read from up to 4 disks in parallel, achieving very high data rates. ${ }^{15}$ For the VMEbus, a system is available that provides a $16 \mathrm{Mbyte} / \mathrm{s}$ data rate, and 7 Mbyte storage capacity. ${ }^{16}$ Such a disk system could be used with a SUN (Sun Microsystems Inc, Mountain View, CA) workstation to build a central buffer with a minimal number of components.

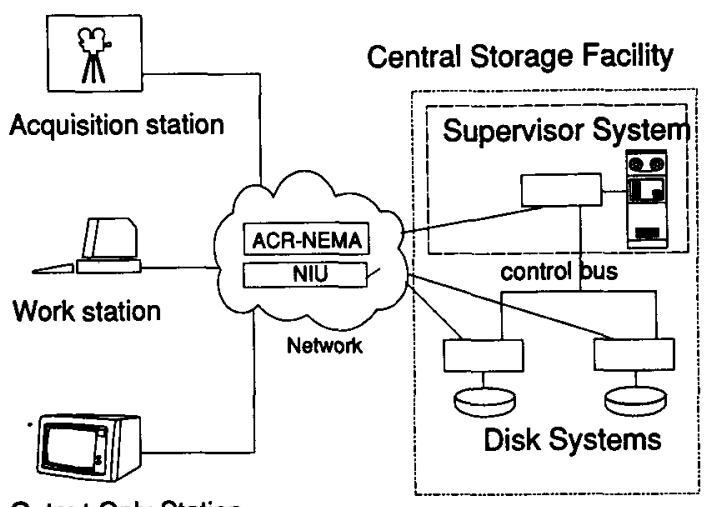

Output Only Station

Fig 5. Alternative PACS system, with a supervisor system to control the archive and multiple (slave) disk systems.

These alternatives have in common with the system presented in the previous sections that their performance will depend critically on the amount of prefetching that can be done from the archive, and on the network protocols that can be used.

\section{CONCLUSIONS AND PERSPECTIVE}

The implementation of a PACS central storage facility is possible with the current technology. From the workload analysis it was concluded that the disk buffer performance is more critical for the overall system performance than archive speed. Both tape and optical archive provide acceptable performance. This conclusion implies that the prefetching strategy is crucial for the overall system performance. A decision between an optical (disk) or magnetic (tape) archive will be based mostly on price and data retention and reliability considerations. Both systems provide acceptable performance for a PACS system.

\section{REFERENCES}

1. Digital Imaging and Communications, American College of Radiology-National Electrical Manufacturers Association standards publication No 300, 1985

2. Cho PS, Huang HK, Tillisch J: Clinical experience with a digital remote viewing station in coronary care unit. Society of Photo-optical Instrumentation Engineers Medical Imaging II 914:1057-1063, 1988

3. Lodder H, Van Poppel BM, De Valk JPJ, et al: HIS-PACS coupling in practice. Society of Photo-optical Instrumentation Engineers Medical Imaging III 1093:301306, 1989

4. Fisher PD, Brauer GW: Impact of image size on effectiveness of digital imaging systems. J Digit Imag 2(1):3941,1989
5. Tape Cartridge Marketing Documentation, Memorex Telex Corporation, Tulsa, OK

6. Gibson GA: Performance and reliability in redundant arrays of inexpensive disks. Computer Measurement Group Inc '89 Proc, Reno, NV, 1989, pp 381-391

7. Comer D: Internetworking with TCP/IP. Englewood Cliffs, NJ, Prentice Hall 1988

8. Katz D: A proposed standard for the transmission of IP datagrams over FDDI networks. RFC-1103, National Science Foundation Network, 1989

9. Greberman M, Mun SK: Image management and communication in patient care: Perspectives on implementation and impact. J Digit Imag 2(1):11-14, 1989

10. Houtekamer GE: Measuring and modelling Disk I/O 
Subsystems. Delft, The Netherlands, Delft University Press, 1989 (ISBN 90-6275-567-4)

11. 3490 Cartridge Subsystem Marketing Materials. IBM Corporation, 1989

12. KODAK Optical Disk System 6800 Automated Disk Library, 1989

13. KODAK Optical Storage Interface-6800-I, 1989
14. Sauer $\mathrm{CH}$, MacNair EA, Kurose JF: The research queueing package Version 2, TSO users guide. IBM Manual No SB11-5898-0, 1984

15. Yager T: Compaq's reason to believe in EISA. Byte 15(3):122-124, 1990

16. Bock M: Parallel disk systems, multiple disk level performance. Computer Technology Review, 1990, pp 41-45 